\title{
A NEW VARIABILITY PARAMETER FOR GAMMA-RAY BURST TIME PROFILES
}

\author{
JOHN PATRICK LESTRADE \\ Department of Physics and Astronomy, Mississippi State University, MS 39762-5167 \\ Received 1994 January 28; accepted 1994 April 11
}

\begin{abstract}
We present a parameter that measures the structure of gamma-ray burst time profiles. This parameter is based on the statistics of runs and is a good measure of time profile variability. It is shown to be independent of burst duration and less sensitive to burst distance than algorithms that depend directly on the intensity of a burst.

Subject heading: gamma rays: bursts
\end{abstract}

\section{INTRODUCTION}

Gamma-ray burst (GRB) time profiles continue to resist efforts of classification and analysis. As shown by Hurley (1991), these profiles show variability on timescales from milliseconds to hundreds of seconds. It is not uncommon in a GRB profile to find peaks riding on other peaks or quiescent periods between peaks where the count rates return completely to the level of the preburst background.

If we can parameterize the observed structure, we may be able to classify bursts or find correlations with other parameters. This in turn could help place constraints on models of the underlying physical phenomena. Of course, there is no one definition of structure. Recently Lamb et al. (1993) used the ratio of the maximum count rates in $64 \mathrm{~ms}$ bins to the maximum in the $1024 \mathrm{~ms}$ bins as a type of burst variability. This definition has the attractive characteristic of applying equally as well to very short bursts as to very long ones. On the other hand it is lacking in that, for any one burst, it does not measure the variability over the complete time history.

In a previous paper (Lestrade et al. 1991), we first presented the idea that a reasonable parameter to measure the variability of a profile is the number of times the profile shows long runs of monotonically increasing (or decreasing) bin-to-bin differences. To the eye, it is the presence of these monotonic runs that is the signature of what we qualitatively call a highly structured burst. The monotonic runs in smooth profiles are short because they are broken up by noise. The statistics of these "runs up," as they are called, although new to the area of GRBs, were heavily studied earlier this century by researchers in agronomy (Wallis \& Moore 1941) and meteorology (Besson 1920). This algorithm has the attractive quality of measuring the structure over the entire history. It has a shortcoming in that for a given data time resolution, there is a duration limit below which there are not enough bins to count runs statistically.

In a recent paper (Lestrade et al. 1994), we applied this statistic to 173 long-duration BATSE GRB profiles. We found no unexpected correlations between structure and other, more easily measurable quantities such as burst angular position, hardness, or intensity. Nor did we find any groupings that would indicate more than one source population. In this Letter, we document the algorithm used and show its lack of dependence on burst duration and burst $V / V_{\max }$.

\section{RUNS UP AND DOWN}

Consider a gamma-ray burst time profile containing $n$ bins of data. Let the number of counts in the ith bin be $b_{i}$. Consider the sequence of signs whose ith element is the sign of $b_{i+1}-b_{i},(i=1,2, \ldots, n-1)$. A subsequence of $p$ consecutive signs (either + or - ) is called a run up (+) or down (-). The notation that we use differs slightly from that used by Knuth (1981) in that, in this Letter, a run of length $p$ involves $p+1$ original bins of data. For example, consider the series of gamma-ray counts registered in the following eight $64 \mathrm{~ms}$ bins: 501, 502, 504, 510, 503, 502, 503, 501. This series contains four runs (up, down, up, down) of lengths 3, 2 , 1 , and 1 , respectively.

Let $r_{p}$ be the number of runs of length $p, r_{p}^{\prime}$ be the number of runs of length $p$ or greater, and $r$ be the total number of runs in a series. Obviously $r_{1}^{\prime}$ equals $r$. Bienayme (1874) was the first to show that the average number of runs in a random series of length $n$ is $\langle r\rangle=(2 n-1) / 3$. The expected values for the number of runs of other lengths are given by Wallis \& Moore (1941) as

$$
\begin{aligned}
& \left\langle r_{p}\right\rangle=2 n \frac{p^{2}+3 p+1}{(p+3) !}-2 \frac{p^{3}+3 p^{2}-p-4}{(p+3) !}, \\
& \left\langle r_{p}^{\prime}\right\rangle=2 n \frac{p+1}{(p+2) !}-2 \frac{p^{2}+p-1}{(p+2) !} .
\end{aligned}
$$

Wallis \& Moore show that a series of data may be tested for randomness by counting the number and lengths of runs. They construct a statistic through the usual procedure of squaring the difference between observed and expected, dividing by the variance and summing these ratios, viz,

$$
\sum_{p=1}^{q-1} \frac{\left(r_{p}-\left\langle r_{p}\right\rangle\right)^{2}}{\sigma^{2}\left(r_{p}\right)}+\frac{\left(r_{q}^{\prime}-\left\langle r_{q}^{\prime}\right\rangle\right)^{2}}{\sigma^{2}\left(r_{q}^{\prime}\right)}
$$


The last term of the sum is an integral term over all runs greater than length $q-1$. However, as they point out, since these runs are not independent, the resultant sums are not distributed according to the usual $\chi^{2}$ statistics. In order to calculate this statistic, we need the variances $\sigma^{2}\left(r_{p}\right)$ and $\sigma^{2}\left(r_{q}^{\prime}\right)$. The formulae for these and other covariances have been provided by Levene \& Wofowitz (1944). They all have the form of $\sigma^{2}=a n+b$ where $n$ is the length of the series. Since $a$ and $b$ are roughly the same magnitude and $n$ is large for our burst profiles, the an term is more important than $b$. Equations (4) and (5) give the forms for $\sigma^{2}\left(r_{p}\right)$ and $\sigma^{2}\left(r_{p}^{\prime}\right)$, respectively, while Table 1 presents the numerical values of $a$ and $b$ for all values of $p$ up to 7 [the seventh values are for the integral term, $\left.\sigma^{2}\left(r_{7}^{\prime}\right)\right]$ :

$$
\begin{aligned}
& \sigma^{2}\left(r_{p}\right)=2 n {\left[-2 \frac{2 p^{5}+15 p^{4}+41 p^{3}+55 p^{2}+48 p+26}{(p+3) !(p+3) !}+2 \frac{2 p^{2}+9 p+12}{(2 p+3)(2 p+5)(p+3) !(p+1) !}\right.} \\
&\left.-4 \frac{4 p^{3}+18 p^{2}+23 p+7}{(2 p+5) !}+\frac{2}{(2 p+1) p ! p !}+\frac{p^{2}+3 p+1}{(p+3) !}\right]+2\left[2 \frac{3 p^{6}+24 p^{5}+69 p^{4}+90 p^{3}+67 p^{2}+42 p+10}{(p+3) !(p+3) !}\right. \\
&\left.-4 \frac{2 p^{3}+11 p^{2}+19 p+9}{(2 p+3)(2 p+5)(p+3) !(p+1) !}+2 \frac{16 p^{4}+80 p^{3}+116 p^{2}+32 p-19}{(2 p+5) !}-4 \frac{p}{(2 p+1) p ! p !}-\frac{p^{3}+3 p^{2}-p-4}{(p+3) !}\right], \\
& \sigma^{2}\left(r_{p}^{\prime}\right)=2 n {\left[-2 \frac{2}{(p+2) !(p+2) !}-\frac{2}{(2 p+3)(p+2) ! p !}-4 \frac{p+1}{(2 p+3) !}+\frac{2}{(2 p+1) p ! p !}+\frac{p+1}{(p+2) !}\right] } \\
&+2\left[2 \frac{(p+1)^{2}\left(3 p^{2}+4 p-3\right)}{(p+2) !(p+2) !}-4 \frac{p}{(2 p+1) p ! p !}+4 \frac{p+1}{(2 p+3)(p+2) ! p !}+2 \frac{4 p^{2}+6 p+1}{(2 p+3) !}-\frac{p^{2}+p-1}{(p+2) !}\right] .
\end{aligned}
$$

It might be thought that the number of runs in a series is dependent on the underlying distribution, for example, uniform or Poissonian. Wallis \& Moore show this not to be the case. While their arguments were based on nonrepeating counts, repetition occurs infrequently enough in our profiles so as to not change their conclusion. Certainly the sizes of the bin-to-bin differences are affected by the statistical distribution, but the signs of the differences (and consequently the number of runs) are not. Our own calculations with Monte Carlo simulations bear this out.

\section{THE PROFILE STRUCTURE PARAMETER, $s$,}

Figure 1 presents the number of runs observed in a $60 \mathrm{~s}$ interval for (1) two BATSE burst profiles (diamond from burst $2 \mathrm{~B} 910425$ at $2265 \mathrm{~s}$ and open circle from burst 2B 920627 at $46,956 \mathrm{~s}$; the latter profile can be seen in Lestrade et al. 1992), (2) BATSE postburst background data (open square), and (3) the expected numbers from equations (1) and (2) (line/dot/error bars). The error bars are the standard deviations from Table 1 . The error bars on the two burst curves are smaller than the diamond and open circle symbols. As shown, the numbers seen in the background agree with the statistical predictions. In a burst profile, however, we see increased numbers of runs for longer lengths $(p>3)$ and usually a smaller number of short runs. It is this difference that we exploit to measure a profile's structure. Following the lead given by equation (3), we have used the expected values and variances to construct a statistic that measures the variability of GRB profiles. The statistic is given by

$$
S_{p}=\sum_{p=4}^{6} \frac{\left(r_{p}-\left\langle r_{p}\right\rangle\right)^{2}}{\sigma^{2}\left(r_{p}\right)}+\frac{\left(r_{7}^{\prime}-\left\langle r_{7}^{\prime}\right\rangle\right)^{2}}{\sigma^{2}\left(r_{7}^{\prime}\right)} .
$$

Note that the $r_{7}^{\prime}$ term accounts for all runs of length 7 or greater. Since we are not strictly trying to measure the deviation from statistical chance, but rather trying to measure the structure of a profile by its frequency of long runs, we sum only over terms larger than $p=3$, and, in addition, we exclude any terms in equation (5) where the observed value is less than the expected value. Not doing so would overinflate the variability parameter when a profile showed less structure than random noise at some value of $p$. A highly structured burst will show more long runs (e.g., $r_{6}, r_{7}$, etc.) than a smooth one leading to a higher value of $S_{p}$. Furthermore, as will be seen below, $S_{p}$ is insensitive to the duration of the burst. Graphically the parameter $S_{p}$ measures how high the burst curve is in Figure 1 above the expected curve for all run lengths greater than $p=3$.

In not counting the shortest runs, our algorithm does miss some short tall "spikes" (i.e., runs that are short in duration but tall in intensity). However, this is not important for two reasons. Figure 2 is a plot of the number of long runs ( $p>3$ ) versus the number of short runs but only for runs whose heights are greater than $5.5 \sigma$. The first thing to notice is that of the 173 profiles only about 40 have more than a single tall spike. The other 133 do not have short (tall) runs and therefore do not appear on the graph. (Because we

TABLE ।

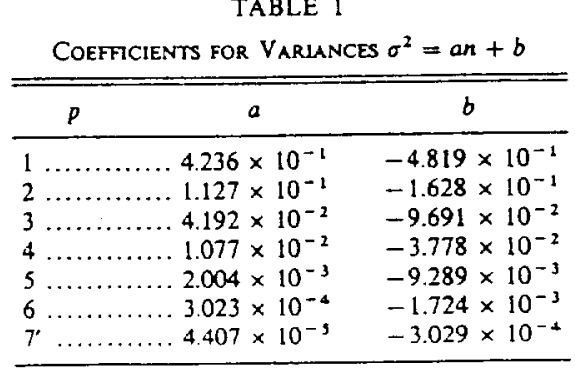






Run Length, $p$



Number of Runs, $p \leq 3$

FiG. 2

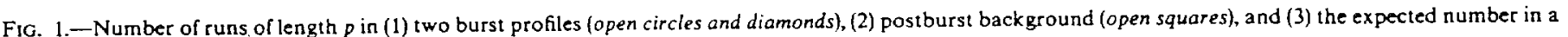
Poissonian background (line/closed circles/error bars) (see text).

FiG. 2.-Number of long runs $(p>3)$ vs. number of short runs $(p \leq 3)$ for runs whose heights exceed $5.5 \sigma$

count runs only within the range of $T_{90}$, we omit the triggering run which is often greater than $5.5 \sigma$.) Second, this figure shows that of the 40 profiles, there is a good correlation between the number of short runs and the number of long ones. Therefore, statistically speaking, the few profiles whose short runs are not counted are also the ones that have the most number of long runs, and so the validity of the parameter is preserved.

\section{THE PEAK HEIGHT PARAMETER, $H$,}

As mentioned above, one of the appealing attributes of $S_{p}$ is that it is not directly dependent on the heights of spikes, that is, the distance to the burster and the intrinsic luminosity function. As a comparison we have constructed a second parameter that is directly dependent on peak heights. Following the lead given by equation (5), we formulate a height parameter given by

$$
H_{p}=\sum_{p=1}^{6}\left(h_{p}-\left\langle h_{p}\right\rangle\right)+\left(H_{p}^{\prime}-\left\langle H_{p}^{\prime}\right\rangle\right) \text {, }
$$

where $h_{p}$ is the sum of heights for all runs of length $p$ in a particular burst profile, $\left\langle h_{p}\right\rangle$ is the height sum observed in a random Poissonian profile, and as above, the sum includes only those values where the observed burst height exceeds the noise profile height. In short, the value of $H_{p}$ is the sum of all spike (or run) heights above that which you would find in a Poissonian profile of the same length.

A single-spike burst (Fast Rise, Exponential-Decay or FRED) with no noise would be composed of two runs (one up and one down). The value of $H$ would then be twice the height of the FRED. Another burst with two equal FREDs would have twice the value of a single FRED. Because of this definition of $H_{p}$, longer bursts will have higher values as will be seen below.

\section{CORRELATION WITH BURST DISTANCE AND DURATION}

Figure 3 shows that both $S_{p}$ and $H_{p}$ depend, to different degrees, on the burst distance or $V / V_{\max }$. The structure parameter, being dependent on the number of runs and not their heights, shows slightly less correlation with distance than does the height parameter. The profiles appearing in these correlations are the same 173 long-duration bursts from Lestrade et al. (1992).

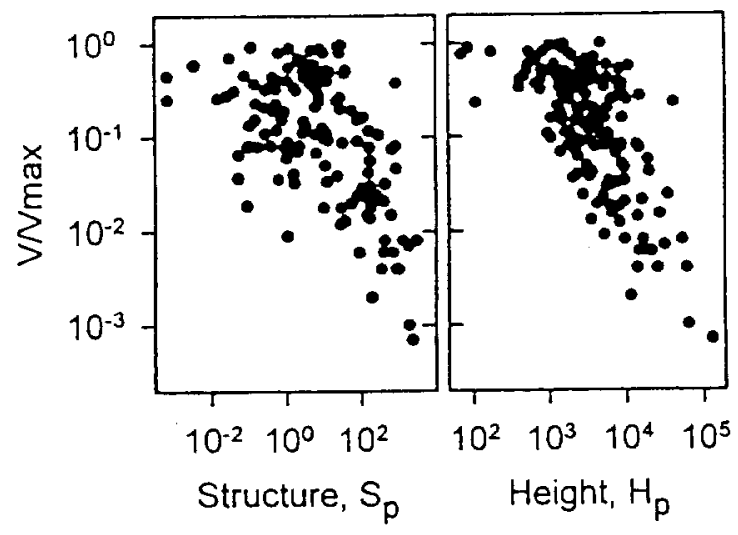

FIG. 3

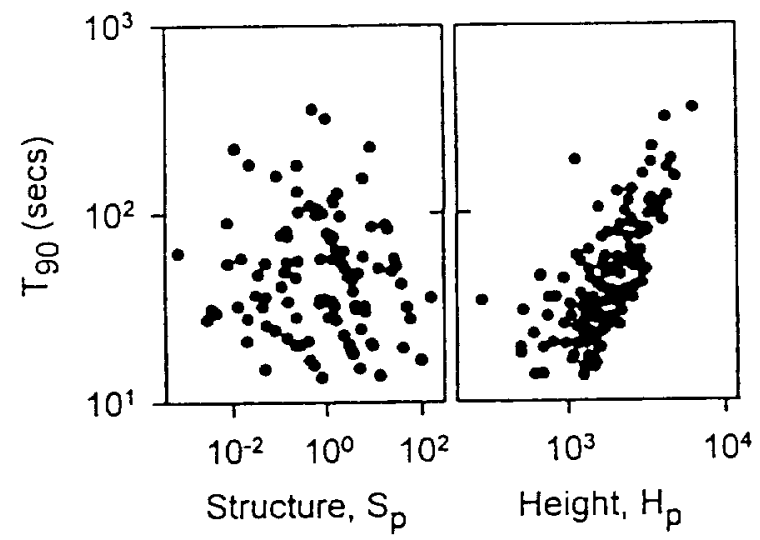

FIC. 4

Fic. 3.-Burst $V / V_{\max }$ vs. $S_{p}$ and $H_{0}$

Fig. 4.-Burst duration vs. $S_{p}$ and $H_{\text {p }}$ for normalized profiles (see text) 
In order to remover the dependence on distance, we normalized the burst profiles by reducing all of them to the same value of $C_{\max }$. The algorithm to handle the reduction and partial addition of Poissonian noise was obtained from Norris \& Nemiroff (1994). Figure 4 presents the graph of duration versus $S_{p}$ and $H_{p}$ for these normalized profiles. As a measure of duration, we use the $T_{90}$ values from Fishman et al. (1994). With the intensities and noise biases rendered uniform, we see very clearly the dependence of $H_{p}$ on duration. The structure $S_{p}$, however, does not show a correlation with burst duration.

\section{CONCLUSION}

The parameter $S_{p}$ is a measure of the variability of a burst profile. It has several properties that make it attractive as a statistic. In addition to being easily formulated and straightforward to calculate, it is independent of background count rate, trigger integration time, and burst duration, and can be made independent of peak intensity. Furthermore, it is less dependent on burst distance than are parameters that depend directly on the heights of profile features.

The author wishes to thank Brad Shaefer, David Palmer, and Jay Norris at GSFC for several helpful comments. This research was supported by NASA grant SUB 92-194.

\section{REFERENCES}

Besson, L. 1920, Monthly Weather Rev., 48, 89

Bienaymé, J. 1874, Bull. de la Socièté Mathématique de France, 2, 153

Fishman, G. J. et al. 1994, ApJS, in press

Hurley, K. 1991, in Gamma-Ray Bursts, ed. W. S. Paciesas \& G. J. Fishman (New York: AIP), 3

Knuth, D. E. 1981, The Art of Computer Programming, Seminumerical Algorithms (2d ed.; Reading Mass.: Addison-Wesley)

Lamb, D. Q., et al. 1993, ApJ, 413 , L11

Lestrade, J. P., et al. 1991, in Gamma-Ray Bursts, ed. W. S. Paciesas \& G. J. Fishman (New York: AIP), 310
Lestrade, J. P., et al. 1992, Compton Gamma-Ray Observatory, ed. M. Friedlander, N. Gehreis, \& D. J. Macomb (New York: AIP), 969

- 1994 in Proc 2d Huntsville Gamma-Ray Burst Conference, ed. K. Hurley G. J. Fishman, \& J. Brainerd (New York: AIP), in press

Levene, H., \& Wolfowitz, J. 1944, Ann. Math. Stat., 15, 58

Norris, J., \& Nemiroff, R. 1994, ApJ, submilted

Wallis, W. A., \& Moore, G. H. 1941, A Significance Test for Time Series (New York: Nat. Bureau of Econ. Res.) 